\title{
A coordination model for ill-posed bilevel programming problem via the leader's motivation.
}

\author{
Wengang Zhang ${ }^{1, a}$, Shihui Jia $^{2, b}$ \\ ${ }^{1}$ Based on the science department, Wuchang Shouyi University, wuhan 430064,china. \\ ${ }^{2}$ Math Department, School of Science, Wuhan University of Science and Technology,Wuhan \\ 430081, China. \\ a8215518@qq.com, ${ }^{b} 40098110 @ q q . c o m$
}

Keywords: Nonlinear programming; III-posed bilevel programming; Coordination model

Abstract. For ill-posed linear bilevel programming problem, this paper studies how the leader motivate the follower to maximize its own profit. Firstly, we present a coordination model. Then, under some conditions, we establish two examples. Finally, numerical results show that the proposed method is feasible.

\section{Introduction}

Bilevel programming plays an important role in different application fields, such as transportation, economics, ecology, engineering and others[1], it has been developed and studied by many authors[2-8]. However, Even a linear bilevel programming problem is generally difficult to be solved and it has been proved to be strongly NP-hard [9].

In this paper, we consider the following linear bilevel programming problem:

$$
" \max _{x \geq 0} c_{1}^{T} x+d_{1}^{T} y
$$$$
\text { s.t. } A_{1} x \leq b_{1}, y \in \psi(x)
$$

where $\psi(x)$ is the set of solutions of the lower level problem,

$$
\begin{gathered}
\max _{y \geq 0} d_{2}^{T} y \\
\text { s.t. } A_{2} x+B_{2} y \leq b_{2} .
\end{gathered}
$$

Here, $x, c_{1} \in R^{n}, y, d_{1}, d_{2} \in R^{m}, A_{1} \in R^{p \times n}, b_{1} \in R^{P}, A_{2} \in R^{q \times n}, B_{2} \in R^{q \times m}, b_{2} \in R^{q}$,

we define $X=\left\{x \in R^{n} \mid A_{1} x \leq b_{1}, x \geq 0\right\}$ and $Y(x)=\left\{y \in R^{m} \mid B_{2} y \leq b_{2}-A_{2} x, y \geq 0\right\}$.

Introducing a profit allocation proportion $\beta$, we present a coordination model of problem (1).Here, $\beta$ is a variable, rather than a parameter. Numerical results show that our method is feasible. We also compare our method with partial cooperation in [4], the results indicate that, although the 
follower in both methods has the same income, the profit of the leader obtained by our method is better than that of Cao and Leung [10].

\section{Coordination problem}

In order to establish theoretical results, we state the main assumptions throughout the paper.

\section{Assumptions:}

(A1)For any $x \in X, Y(x) \neq \phi$, there exists a compact subset $W \subseteq R^{m}$ such that $Y(x) \subset W$ for all $x \in X$.

(A2)The set $X$ is a bounded polyhedron.

Since the leader can observe his/her best profit only under the optimistic formulation, he/she can design an incentive based strategy. That is, apart from individual income, he/she can pay the follower a fraction $(1-\beta)$ of the observable profit. This strategy will not only motivate the follower to cooperate with the leader, but also, the leader can choose the profit allocation proportion $\beta$ in such a way his/her part of the profit is maximized. In this paper,we consider the following coordination problem of problem (1)-(2):

$$
\begin{aligned}
& \text { s.t. } x \in X, y \in \psi(x) \\
& \max _{x, y, \beta} \beta\left(c_{1}^{T} x+d_{1}^{T} y\right) \\
& 0 \leq \alpha \leq \beta \leq 1 \\
& d_{2}^{T} y+(1-\beta)\left(c_{1}^{T} x+d_{1}^{T} y\right) \geq d_{2}^{*}
\end{aligned}
$$

Where $\alpha$ is a parameter and $d_{2}^{*}=\max \left\{d_{2}^{T} y \mid x \in X, y \in Y(x)\right\}$.

Without loss of generality, let $\alpha=0.7$ and constraint (4) means the leader may pay the follower 30 percent of the observable profit at most. The proportion is reasonable because the leader is dominant in the decision-making. Of course, we may adjust it according to practical requirements. Constraint (5) guarantees that the follower can get a maximum income $d_{2}^{*}$ regardless of what the leader's choice is, and then, will cooperate with the leader. Moreover, if $(\bar{x}, \bar{y}, \bar{\beta})$ is a solution of problem (3), the follower will receive a fraction $(1-\bar{\beta})$ of the leader's profit apart from individual income $d_{2}^{T} \bar{y}$. And the leader's profit is $\bar{\beta}\left(c_{1}^{T} \bar{x}+d_{1}^{T} \bar{y}\right)$. 


\section{Numerical Results}

In this section, we consider the following examples which are from [10].

Example 1: $\quad$ " $\max _{x} " 8 x_{1}+10 x_{2}+2 y_{1}-y_{2}$

$$
\text { s.t. } x_{1}+x_{2} \leq 10, x_{1}, x_{2} \geq 0
$$

where $y=\left(y_{1}, y_{2}\right)^{T}$ solves: $\max _{y} y_{1}+y_{2}$

$$
\begin{aligned}
& \text { s.t. } y_{1}+y_{2} \leq 20+x_{1}-x_{2}, \\
& y \geq 0 .
\end{aligned}
$$

Example 2: $\quad$ " $\max _{x} " 8 x_{1}+6 x_{2}+25 y_{1}+30 y_{2}-2 y_{3}-16 y_{4}$

$$
\text { s.t. } x_{1}+x_{2} \leq 10, x_{2}, x_{2} \geq 0 \text {, }
$$

where $y=\left(y_{1}, y_{2}, y_{3}, y_{4}\right)^{T}$ solves: $\max _{y} 10 y_{1}+10 y_{2}+10 y_{3}+10 y_{4}$

$$
\begin{array}{ll}
\text { s.t } & y_{1}+y_{2}+y_{3}+y_{4} \leq 10-x_{1}-x_{2}, \\
& -y_{1}+y_{4} \leq 0.8 x_{1}+0.8 x_{2}, y_{2}+y_{4} \leq 4 x_{2}, y \geq 0 .
\end{array}
$$

For solving problem (3),we choose $k_{1}=k_{2}=10, \gamma=10$ and $\alpha=0.7$. The numerical results are reported in Table 1, where $L$ and $F$ denote the profit of the leader and the follower respectively.Moreover,the proposed method in this paper is denoted by Method 1, and the method of Cao and Leung [10] by Method 2.

Method 1

Method 2

$$
(x, y)^{T} \quad L \quad F \quad \beta \text { (proportion) } \quad x^{T} \quad L \quad F \quad \lambda \text { (cooperation degree) }
$$

Ex1 $(10,0,30,0)^{T} \quad 140 \quad 30 \quad 1 \quad(10,0)^{T} \quad 140 \quad 301$

Ex2 $(0,2,0,8,0,0)^{T} 232 \quad 100 \quad 0.9206 \quad(0,0)^{T} \quad 185.7 \quad 100 \quad 0.762$ 
For example 1, the optimistic solution is $(10,0)^{T}$. The optimistic value is 140 . The result of our method is equal to that of [4]. And they are also equal to that of the optimistic formulation.

For example 2, $(0,2)^{T}$ is an optimistic solution with optimistic value 252. Method 1 and method 2 have the same revenues for the follower. However, the leader's return of the former is more than that of the latter. These mean that, our method is superior to the method of Cao and Leung [10]. The reason is that, except for guaranteeing the maximization of the follower's interest, in [10], the leader's return depends on the partial cooperation degree $\lambda$ of the follower. This dependency is also reflected in their partial cooperation model, which is solved for a range of $\lambda$ values, That is, only after given a value of parameter $\lambda$ can the model be solved. It will undoubtedly improve and enhance the status of the follower in the decision making, and even turns the previous leader-follower relation upside down.

Note that, in our method, the leader's return also depends on the profit allocation proportion $\beta$. However it is a variable, and plays a major role in maximizing the leader's profit potential although it is affected by the follower. At this point, the leader still plays a primary role in the decision making which is different from that of [10].

\section{Conclusion}

In this paper, we present a coordination model for ill-posed linear bilevel programming problem. It can motivate the follower to cooperate with the leader, and then maximize the leader's interests. Numerical results show that the proposed method is feasible, and the leader can maximize his/her interests by motivating the follower. For the future research, it is interesting and useful to consider a general coordination model of ill-posed nonlinear bilevel programming problem.

\section{References}

[1]S.Dempe.Annotated bibliography on bilevel programming and mathematical programs with equilibrium constrains[J], Optimization, 2003,52:333-359.

[2]J.F.Bard. Practical Bilevel Optimization:Algorithms and Applications[M], Kluwer Academic Publishers, Dordrecht, 1998.

[3]B.Colson,P.Marcotte and G.Savard. An overview of bilevel optimization [J]. Annals of Operations Research, 2007,153: 235-256.

[4]S.Dempe.Foundations of bilevel programming[M].Kluwer Academic Publishers,2002.

[5]K.Shimizu,Y.Ishizuka and J.F.Bard.Nondifferentiable and two-level mathematical programming [M].Kluwer Academic,Dordrecht,1997. 
[6]L.Vicente.and P.Calamai.Bilevel and multibilevel programming: a bibliography review [J], Journal of Global Optimization, 1994,5: 291-306

[7]U.Wen.and S.Hsu.Linear bilevel programming problem- a review[J],Journal of the Operational Research Society, 1991, 42(2):125-133.

[8]Y.Zheng and Z.Wan, A solution method for semivectorial bilevel programming problem via penalty method[J]. Journal of Applied Mathematics and Computing. 2011,37(1-2),207-219.

[9]P.Hansen.,B.Jaumard.and G.Savard.New branch-and-bound rules for linear bilevel programng [J]. SIAM Journal on Science and Statistical Computing,1992,13:1194-1217.

[10]D.Cao.and L.Leung. A partial cooperation model for non-unique linear two-level decision problems[J].European Journal of Operation Research.140:134-141(2002) 